\title{
Causal vs. Noncausal Description of Nonlinear Wave Mixing; Resolving the Damping-Sign Controversy
}

\author{
Shaul Mukamel \\ Department of Chemistry, University of California, Irvine, CA 92697
}

(Dated: December 22, 2018)

\begin{abstract}
Frequency-domain nonlinear wave mixing processes may be described either using response functions whereby the signal is generated after all interactions with the incoming fields, or in terms of scattering amplitudes where all fields are treated symetrically with no specific time ordering. Closed Green's function expressions derived for the two types of signals have different analytical properties. The recent controversy regarding the sign of radiative damping in the linear (Kramers Heisenberg) formula is put in a broader context.

Submitted to Phys. Rev. A (Rapid communication)

PACS Indices

42.65.An, 32.80.-t, 42.50.ct, 32.70.Jz
\end{abstract}




\section{INTRODUCTION}

A lively debate is currently going on with regard to the correct sign that should be introduced in the Kramers-Heisenberg expression for the (linear) optical polarizability. Both "opposite sign" and a "constant sign" formulas have been derived by various authors. Perturbative QED calculations have been carried out to include radiative damping [1-8]. In an insightful recent article Bialyncki-Birula and Sowinski [9] had pointed out that this issue is fundamentally connected with the linear response vs. the scattering points of view for Rayleigh scattering. In this letter we extend this argument to nonlinear wave mixing of arbitrary order. Closed formal expressions are derived that reveal the analytical properties of both retarded (response) and non retarded (scattering) signals. The linear response results are recovered to lowest order. Our microscopic deviation could serve as the starting point for a full QED calculation. However, this is not required in order to pinpoint the analytical properties which should hold for other types of nonradiative damping as well.

The following derivation applies for processes of arbitrary order. However, for clarity we focus on four wave mixing. Consider a system (atom, molecule) interacting with four modes of the radiation field. The states of the system will be denoted a,b,c... . The j'th mode of the radiation field has a frequency $\omega_{j}$ and initial occupation number $n_{j}$. To simplify the notation we hereafter consider sum frequency generation whereby $\omega_{4}=\omega_{1}+\omega_{2}+\omega_{3}$, however the results can be easily extended to any combination of frequencies $\omega_{4}= \pm \omega_{1} \pm \omega_{2}+ \pm \omega_{3}$. For the process of interest the initial state of the system + field is $|i>=| a, n_{1} n_{2} n_{3} n_{4}>$, and the final state is $|f>=| a, n_{1}-1, n_{2}-1, n_{3}-1, n_{4}+1>$, with energies $E_{i}$ and $E_{f}$ respectively.

The coupling of an atom located at point $\mathbf{r}$ with the radiation field is

$$
H_{\text {int }}=-V \cdot E(\mathbf{r}, t)
$$

We shall divide the electric field into its positive and negative frequency components

$$
\begin{gathered}
E(\mathbf{r}, t)=\varepsilon(\mathbf{r}, t)+\varepsilon^{\dagger}(\mathbf{r}, t) \\
\varepsilon(\mathbf{r}, t)=\sum_{j}\left(\frac{2 \pi \hbar \omega_{j}}{\Omega}\right)^{1 / 2} \exp \left(i k_{j} \mathbf{r}-i \omega_{j} t\right) a_{j}
\end{gathered}
$$


$a_{j}$ is the photon annihilation operator of mode $j, \mathrm{~V}$ is the dipole operator and $\Omega$ is the quantization volume.

We first assume that the system is prepared in a nonequilibrium steady state with the four fields and consider the entire process as a single four-photon scattering event. This is a non causal process whereby all four modes are treated along the same footing. This implies that the interaction with model 4 need not be the last, and all time orderings are allowed and should be summed over. The process is described by the S matrix element

$$
S_{f i}=A_{f i} T_{f i}\left(E_{i}\right) \delta\left(E_{i}-E_{f}\right),
$$

where $T_{f i}$ is the matrix element of the $\mathrm{T}$ matrix $\mathrm{T}=\mathrm{V}+\mathrm{V} \mathrm{G}(\mathrm{E}) \mathrm{V}$, and

$$
G(E)=\frac{1}{E-H+i \varepsilon}
$$

is the retarded Green's function. The scattering amplitude for this process is given by

$$
\begin{gathered}
S_{f i}^{(4)}=A_{f i} \sum_{p_{4}} \\
<a\left|V G\left(E_{a}+\omega_{1}+\omega_{2}+\omega_{3}\right) V G\left(E_{a}+\omega_{1}+\omega_{2}\right) V G\left(E_{a}+\omega_{1}\right) V\right| a>\delta\left(\omega_{1}+\omega_{2}+\omega_{3}-\omega_{4}\right)
\end{gathered}
$$

where

$$
A_{f i}=\left(\frac{2 \pi \hbar}{\Omega}\right)^{2} \sqrt{n_{1} n_{2} n_{3}\left(n_{4}+1\right)} \sqrt{\omega_{1} \omega_{2} \omega_{3} \omega_{4}}
$$

$\sum_{p_{4}}$ denotes the sum over all 4 ! permutations of $\omega_{1}, \omega_{2}, \omega_{3}$, and $-\omega_{4}$. The sign convention for $\omega_{j}$ in eq. 6 is as follows: an absorbed photon gives $+\omega_{j}$ whereas an emitted photon gives $-\omega_{j}$. In the process considered here, $\omega_{1}, \omega_{2}$ and $\omega_{3}$ are absorbed and $\omega_{4}$ is emitted. Other processes can be calculated by simply changing the signs of $\omega_{j}$, as warranted in each case. $\mathrm{H}$ is the Hamiltonian for the atom + all modes of the radiation field excluding the 4 modes of interest, since those were treated perturbatively.

We next turn to the standard semiclassical description of four wave mixing whereby the system first interacts with modes 1,2 and 3 to generate the third-order polarization which then serves as a source for mode 4 [10]. This is a causal response where mode 4 is special 
since its interaction with the system must be the very last. We shall calculate the n'th order polarization as the expectation value of $\mathrm{V}[11]$

$$
P^{(n)}=\sum_{m=0}^{n}<\Psi^{(m)}|V| \Psi^{(n-m)}>,
$$

where $\Psi^{(m)}$ is the perturbed wavefunction to $m^{\prime}$ th order in $V$. The $m^{\prime} t h$ term represents $(n-m)^{\prime}$ th order for the ket and $m^{\prime}$ th order for the bra. Overall there are $(n+1)$ terms. For $n=3$ we get four terms corresponding to $m=0,1,2,3$ respectively in eq.(8). We then get $P^{(3)}=A_{f i} \chi_{f i}^{(3)}$ where

$$
\begin{aligned}
\chi_{f i}^{(3)}= & \sum_{p_{3}} \\
< & a\left|V G\left(E_{a}+\omega_{1}+\omega_{2}+\omega_{3}\right) V G\left(E_{a}+\omega_{1}+\omega_{2}\right) V G\left(E_{a}+\omega_{1}\right) V\right| a> \\
< & a\left|V G^{\dagger}\left(E_{a}+\omega_{1}+\omega_{2}-\omega_{4}\right) V G\left(E_{a}+\omega_{1}+\omega_{2}\right) V G\left(E_{a}+\omega_{1}\right) V\right| a> \\
+< & a\left|V G^{\dagger}\left(\omega_{1}-\omega_{4}+\omega_{2}\right) V G^{\dagger}\left(E_{a}+\omega_{1}-\omega_{4}\right) V G\left(E_{a}+\omega_{1}\right) V\right| a> \\
+< & a\left|V G^{\dagger}\left(E_{a}-\omega_{4}+\omega_{1}+\omega_{2}\right) V G^{\dagger}\left(E_{a}-\omega_{4}+\omega_{1}\right) V G^{\dagger}\left(E_{a}-\omega_{4}\right) V\right| a> \\
& \delta\left(\omega_{1}+\omega_{2}+\omega_{3}-\omega_{4}\right),
\end{aligned}
$$

is the susceptibility and

$$
G^{\dagger}(E)=\frac{1}{E-H-i \varepsilon}
$$

is the advanced Green's function. $\quad \sum_{p_{3}}$ denotes the sum over all 3! permutations of the incoming modes $\omega_{1}, \omega_{2}$ and $\omega_{3}$. In this expression the sign conversion of $\omega_{j}$ is the same as in eq. (6) (+sign for absorbed photons, - for emitted). Each coupling with the ket is accompanied by a retarded Green's function $G$, whereas an advanced Green's function $G^{\dagger}$ is accompanied by bra interactions. Eq. (8) will thus yield $(n-m) G$ and $m G^{\dagger}$ factors. An analogous expression was obtained recently using superoperators in Liouville Space. [12] The present Hilbert Space form is more suitable for comparison with the scattering amplitudes.

The extension of Eqs.(6) and (9) to arbitrary order is straightforward. $S^{(n+1)}$ will have a single basic term with $(n+1)$ V factors, and $n$ retarded Green's functions $G$ with arguments $E_{a}+\omega_{1}, \ldots E_{a}+\omega_{1}+\omega_{2}+\ldots+\omega_{n .}$. In addition, it contains a sum over the $(n+1)$ ! permutations of $\omega_{1}, \ldots, \omega_{n+1}$. Each $\omega_{j}$ may be changed to $-\omega_{j}$ to describe different processes. $\chi^{(n)}$ has 
$(n+1)$ basic terms each containing $(n+1) \mathrm{V}$ factors, and a $\left(G^{+}\right)^{m}(G)^{n-m}$ factor with $m=0, \ldots n$ (See Eq.8). The retarded Green's functions will only depend on the incoming frequencies $\omega_{1}, \ldots, \omega_{n}$. All advanced Green's functions also depend on the signal frequency $-\omega_{n+1}$. Each basic term yields $n$ ! terms upon the permutation over $\omega_{1} . . \omega_{n}$. Altogether both $S^{(n+1)}$ and $\chi^{(n)}$ contain $(n+1)$ ! terms. For $S^{(n+1)}$ these come from the $(n+1)$ ! permutations of the $(n+1)$ frequencies over a single term. $\chi^{(n)}$ has $(n+1)$ basic terms, each containing $n$ ! permutations of the $n$ "incoming" frequencies. Unlike $\chi^{(n)}, S^{(n+1)}$ is symmetric with respect to all $(n+1)$ modes; the interaction with the signal field $\omega_{n+1}$ need not be chronologically the last. $S^{(n+1)}$ only contains retarded Green's functions and all propagations are forward in time. The $(n+1)$ ! permutations take care of the possible time orderings of interactions with the various modes. $\chi^{(n)}$, in contrast, depends on both retarded and advanced Green's functions which correspond to forward and backward propagations respectively along the Keldysh Schwinger loop [13-15].

Both expressions (6) or (9), can serve as a starting point for a full QED perturbative calculation, where the coupling with all modes of the electromagnetic field is included in the Hamiltonian H. This will result in damping terms. However, the analytical properties of the two signals are completely determined by eqs (6) or (9), and will be invariant to the level of approximation used for the radiative damping. When all frequencies are tuned off resonance, we can neglect the $\pm i \varepsilon$ terms in the Green's functions, setting $G=G^{\dagger}$. The causal and non causal expressions are then identical.

The ongoing damping sign controversy was restricted to the linear response of a two level system with ground state a and an excited state b [1-9]. This may be immediately resolved by our general formulation. For Rayleigh (elastic) scattering we have one absorbed and one emitted photon with the same frequency. We thus set $\omega_{1}=\omega$ and $\omega_{2}=-\omega$. The second order analogue of eq.(6) reads

$$
S_{f i}^{(2)} \sim<a\left|V G\left(E_{a}+\omega\right) V\right| a>+<a\left|V G\left(E_{a}-\omega\right) V\right| a>
$$

This is obtained from a single term +2 permutations of $\omega$ and $-\omega$. Writing the matrix elements explicitly for the system Hamiltonian (neglecting coupling with other radiation modes), this gives

$$
S_{f i}^{(2)} \sim\left|V_{a b}\right|^{2}\left[\frac{1}{E_{a}+\omega-E_{b}+i \varepsilon}+\frac{1}{E_{a}-\omega-E_{b}+i \varepsilon}\right] .
$$


This is known as the "constant sign" prescription (both terms have a $+i \varepsilon$ factor) [1-9]. The linear response expression similar to eq.(9) gives on the other hand

$$
\chi_{f i}^{(1)} \sim<a\left|V G\left(E_{a}+\omega\right) V\right| a>+<a\left|V G^{\dagger}\left(E_{a}-\omega\right) V\right| a>
$$

This comes from two basic terms with no permutation. (there is only one incoming field which is absorbed and has frequency $\omega$ ). Taking the matrix elements we recover the "opposite sign" prescription

$$
\chi_{f i}^{(1)} \sim\left|V_{a b}\right|^{2}\left[\frac{1}{E_{a}+\omega-E_{b}+i \varepsilon}+\frac{1}{E_{a}-\omega-E_{b}-i \varepsilon}\right] .
$$

The origin of the different damping signs of $S^{(2)}$ and $\chi^{(1)}$ had been clearly pointed out by Bialynicki-Birulya and Sowinski [9] who had further carried out a fourth order QED calculation of dampling using both expressions. The present results extend these arguments to nonlinear processes of arbitrary order. It should be emphasized that the fundamental principle of causality must always hold and is never in doubt. The terms "causal" and "noncausal" in this letter refer to different observables. Traditional semiclassical formulation

of nonlinear optics imposes a certain time ordering by singling out one of the fields. This response is causal. The scattering description is noncausal since it allows for arbitrary time ordering of interactions with the various fields.

The support of the National Science Foundation (Grant No. CHE-0446555) and NIRT (Grant No. EEC0303389) is gratefully acknowledged. I wish to thank Professor Bialynicki Birula for useful discussions.

\section{References}

1. D.L. Andrews, S. Naguleswaran, and G.E. Stedman, Phys. Rev. A 57, 4925 (1998).

2. A.D. Buckingham and P. Fischer, Phys.Rev. A 61, 35801 (2000).

3. A.D. Buckingham and P. Fischer, Phys.Rev. A 63, 47802 (2001).

4. G.E. Stedman, S. Naguleswaran, D.L. Andrews, and L.C. Davila Romero, Phys. Rev. A 63, 47801 (2003).

5. D.L. Andrews, L.C. Davila Romero and G.E. Stedman, Phys. Rev. A 67, 55801 (2003).

6. $\quad$ P.W. Milonni and R.W. Boyd, Phys. Rev. A 69, 23814 (2004).

7. $\quad$ P.R. Berman, R.W. Boyd and P.W. Milonni, Phys. Rev. A 74, 53816 (2006). 
8. R. Loudon and S.M. Barnett. J. Phys. B: At. Mol. Opt. Phys. 39, S555 (2006).

9. I. Bialynicki-Birula and T. Sowinski, Quantum electrodynamics of qubits, arXiv:0705.2121v1.

10. N. Bloembergen, Nonlinear optics (Benjamin, New York, 1965).

11. S. Mukamel, Principles of Nonlinear Spectroscopy (Oxford University Press, New York, 1995).

12. S. Mukamel, Partially-Time-Ordered Keldysh-Loop Expansion of Coherent Nonlinear Optical Susceptibilities, Submitted to Phys. Rev. A. (2007).

13. L. V. Keldysh, Sov. Phys. JETP 20, 1018 (1965).

14. R. Mills, Propagators for many-particle systems; an elementary treatment (New York, Gordon and Breach 1969).

15. H. Haug and A-P. Jauho, Quantum Kinetics in Transport and Optics of Semiconductors (Springer-Verlag, Berlin, Heidelberg, 1996). 